\title{
A requirement engineering model for big data software
}

\begin{abstract}
Most prevailing software engineering methodologies assume that software systems are developed from scratch to capture business data and subsequently generate reports. Nowadays, massive data may exist even before software systems are developed. These data may also be freely available on Internet or may present in silos in organizations. The advancement in artificial intelligence and computing power has also prompted the need for big data analytics to unleash more business values to support evidence-based decisions. Some business values are less evident than others, especially when data are analyzed in silos. These values could be potentially unleashed and augmented from the insights discovered by data scientists through data mining process. Data mining may involve overlaying and merging data from different sources to extract data patterns. Ideally, these values should be eventually incorporated into the information systems to be. To realize this, we propose that software engineers ought to elicit software requirements together with data scientists. However, in the traditional software engineering process, such collaboration and business values are usually neglected. In this paper, we present a new requirement engineering model that allows software engineers and data scientists to discover these values hand in hand as part of software requirement process. We also demonstrate how the proposed requirement model captures and expresses business values that unleashed through big data analytics using an adapted use case diagram.
\end{abstract}

Keyword: Big data requirement; Requirement model; Actionable intelligence; Big data system; Data scientist model 